\title{
Review
}

Cerebrovascular

Diseases

\section{Latin American Consensus Statement for the Use of Contrast-Enhanced Transcranial Ultrasound as a Diagnostic Test for Detection of Right-to-Left Shunt}

\author{
Viviane Flumignan Zetola ${ }^{a}$ Marcos Christiano Lange ${ }^{a}$ Valeria Cristina Scavasine ${ }^{a}$ \\ Rodrigo Bazan ${ }^{b}$ Gabriel Pereira Braga ${ }^{c}$ Ana Claudia C.B. Leite ${ }^{d}$
}

Soraia R. Cabette Fabio ${ }^{e}$ Octavio Marques Pontes Neto ${ }^{e}$

Carla Heloisa Cabral Moro ${ }^{f}$ Gisele Sampaiog ${ }^{g}$ Edson Bor-Seng Shu ${ }^{g}$

Marcelo de Lima Oliveirag Maramelia A. de Miranda Alves ${ }^{\text {h }}$

Daniela Laranja Gomes ${ }^{h}$ Pedro Antonio Pereira de Jesus ${ }^{i}$ Jamary Oliveira-Filho ${ }^{i}$

Wagner Mauad Avelar' Gabriel R. de Freitask ${ }^{\mathrm{j}}$ Rafael Borsoi'

${\text { Corina } \text { Puppo }^{m} \text { Silvia Viviana Cocorullo }}^{n}$ Alejandro Brunser ${ }^{\circ}$ Ayrton Massaro $^{p}$

Natan M. Bornstein ${ }^{q}$ Alexander Razumovsky ${ }^{r}$ Massimo Del Sette ${ }^{s}$

a Division of Neurology, Hospital de Clínicas, Federal University of Parana, Curitiba, Brazil; b Division of Neurology, School of Medicine of Botucatu, São Paulo State University "Júlio de Mesquita Filho", Botucatu, Brazil; 'Division of Neurology, Maria Aparecida Pedrossian University Hospital, Federal University of Mato Grosso do Sul, Campo Grande, Brazil;

${ }^{d}$ State Institute of Hematology Arthur de Siqueira Cavalcanti, Rio de Janeiro, Brazil; e Division of Neurology, Ribeirão Preto Medical School, University of São Paulo, Ribeirão Preto, Brazil; ${ }^{\mathrm{f} D i v i s i o n}$ of Neurology, Hospital Municipal Sao Jose, University of Joinville Region, Joinville, Brazil; ${ }^{9}$ Division of Neurology, Hospital Israelita Albert Einstein, Federal

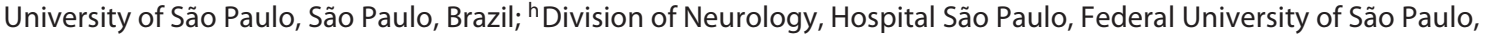
São Paulo, Brazil; 'Cardiomyopathy Clinic, Federal University of Bahia, Salvador, Brazil; 'ंDepartment of Neurology,

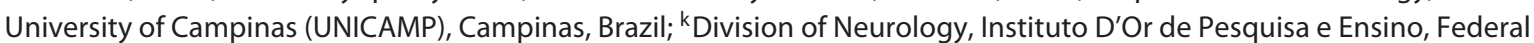
Fluminense University, Rio de Janeiro, Brazil; 'Cardiopulmonary Unit, Hospital de Clínicas, Echocardiographic Service, Federal University of Parana, Curitiba, Brazil; ${ }^{\mathrm{m}}$ Centro de Tratamento Intensivo, Hospital de Clínicas, Universidad de la República, Montevideo, Uruguay; ${ }^{\mathrm{n}}$ Division of Neurology, University of Buenos Aires, Buenos Aires, Argentina; ${ }^{\circ}$ Division

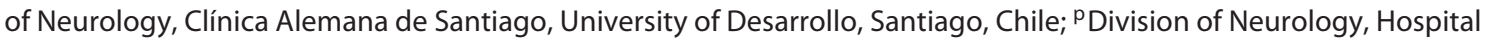

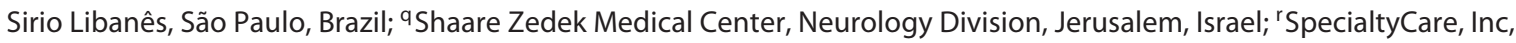

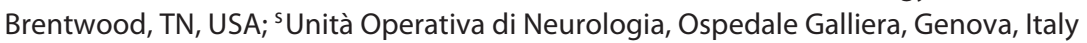

\section{Keywords}

Transcranial Doppler · Contrast-transcranial Doppler . Patent foramen ovale $\cdot$ Right to left shunt $\cdot$ Statement

\begin{abstract}
Background: The role of patent foramen ovale is a field of debate and current publications have increasing controversies about the patients' management in young undetermined stroke. Work up with echocardiography and transcra-
\end{abstract}

\section{KARGER}

(c) 2019 S. Karger AG, Basel

E-Mail karger@karger.com

www.karger.com/ced
Viviane Flumignan Zetola

Flumignano Institute of Medicine

Division of Vascular Neurology, Federal University of Parana

711 Atilio Borio St Alto da Rua XV, PR 80040-120 Curitiba (Brazil)

E-Mail viviane.zetola@gmail.com 
nial Doppler (TCD) can aid the decision with better anatomical and functional characterization of right-to-left shunt (RLS). Medical and interventional strategy may benefit from this information. Summary: a group of experts from the Latin American participants of the Neurosonology Research Group (NSRG) of World Federation of Neurology created a task force to review literature and describe the better methodology of contrast TCD (c-TCD). All signatories of the present consensus statement have published at least one study on TCD as an author or co-author in an indexed journal. Two meetings were held while the consensus statement was being drafted, during which controversial issues were discussed and voted on by the statement signatories. The statement paper was reviewed and approved by the Executive Committee of the NSRG of the World Federation of Neurology. The main objective of this consensus statement is to establish a standardization of the C-TCD technique and its interpretation, in order to improve the informative quality of the method, resulting in expanding the application of TCD in the clinical setting. These recommendations optimize the comparison of different diagnostic methods and encourage the use of C-TCD for RLS screening and complementary diagnosis in multicenter studies.

(c) 2019 S. Karger AG, Basel

\section{Introduction}

The reason to issue this consensus statement is the need for technical standardization of contrast transcranial Doppler (c-TCD) examination for the diagnosis of right-to-left shunt (RLS), with the ultimate aim to improve the reliability of the diagnostic method while maintaining sensitivity and specificity of the test. In addition to these important principles, which are the prerogative of any complementary examination, the publication of this guideline has the benefit of facilitating the comparison of diagnostic methods and encouraging the use of this complementary tool for RLS screening in multicenter studies. Diagnosis of paradoxical embolism and determination of stroke etiology carries great importance, as it may result in different therapeutic strategies [1-5]. Epidemiological and animal studies have suggested that there is an intricate relationship between RLS and cryptogenic stroke and subclinical deep white matter hyperintensities [4]. It is important to remember, however, that paradoxical embolism is not the only mechanism for stroke in patients with patent foramen ovale (PFO); Thrombi in atrial septal aneurysm, left atrial dysfunction and supraventricular arrhythmia may also play a role $[6$,
7]. The adequate use of tools for the diagnosis and characterization of RLS is essential for risk stratification and elucidating other clinical questions. For the purposes of this consensus statement, RLS is considered to be due to a defect of the cardiac interatrial septum named PFO. The literature search report that formed the basis of the present consensus statement is also provided, in order to ensure the understanding of the guidelines by experts of other specialties.

All signatories of the present consensus statement have published at least one study on TCD as an author or co-author in an indexed journal. Two meetings were held while the consensus statement was being drafted, during which controversial issues were discussed and voted on by the statement signatories. The statement paper was reviewed and approved by the Executive Committee of the Neurosonology Research Group of the World Federation of Neurology.

\section{Literature Review}

PFO is best defined as a space between the septum primum and septum secundum, which may be present in $20-30 \%$ of the general population; this may generate such hemodynamic conditions in the atria that allow bidirectional flow $[8,9]$. RLS-related paradoxical embolism is a possible cause of stroke of unknown etiology, particularly among younger patients. Establishing an etiological association or an incidental finding is a difficult task. The diagnostic strategy typically involves an extensive list of complementary examinations (ASCOD phenotype classification - atherosclerosis, small-vessel disease, cardiac pathology, other cause, dissection) [10]. The aim is to obtain a characterization of the functional and anatomical aspects of PFO that may explain the relationship between transient clinical presentations that are directly related to a cardiac event. The dilemma consequently extends to determining the most suitable therapeutic option, which has been the objective of several publications and controversies among researchers $[1-3,5,11-13]$. The establishment of stroke etiology is a dynamic process that involves even the use of recently developed technology, such as the implantable cardiac event monitors; yet, such methods often do not provide sufficient data by themselves and the diagnostic approach depends on each clinical scenario. Indeed, approximately $25-45 \%$ of ischemic strokes are diagnosed as being of an undetermined source [14].

The risk of RLS-related paradoxical embolism is evaluated using several risk assessment tools $[15,16]$ and defi- 
nitions [10] aiming to facilitate the identification of highrisk patients. The use of demographic characteristics, such as age, clinical presentation (and cortical lesions), association with other abnormalities of the atrial septum (such as atrial septal aneurysm), and evaluation of the clinical picture seem insufficient for decision making; thus, the effectiveness of considering such associations remains controversial. The search for accurate predictors is still ongoing, even in the face of current publications [17].

Randomized controlled studies offer the best means to assess the safety and efficacy of percutaneous closure and antithrombotic therapy for PFO treatment. The last 3 and recent studies have demonstrated the superiority of percutaneous closure in preventing the recurrence of vascular events in patients aged 18-60 years with transient ischemia or stroke of unknown etiology [18]. However, considering the different inclusion criteria, disease classification, time to close, stroke workup, diagnostic tools, occluder devices type, duration of follow-up and biases, stronger evidence is needed before recommending the indication for percutaneous closure in clinical practice for all patients. Studies have been limited by the low rate of recurrence of vascular events. In fact, a recent meta-analysis comparing percutaneous closure versus medical therapy for stroke with PFO showed a number needed to treat of 39 for recurrent stroke [19]. Published series including post-occlusion data revealed different outcomes for each of the 4 types of closure devices. Specifically, some devices provided better success rates and reduced incidence of complications [20,21], raising significant doubts regarding the indication for a specific closure device without careful prior assessment. Attention is needed in the analysis of these 3 new published studies. Only the CLOSE trial tested more them one PFO occluder device and was the only one that explored the use of anticoagulant. The authors even mentioned that the effects of this treatment therapy on the risk of stroke recurrence could not be determined [1]. Meanwhile, a subgroup analysis of NAVIGATE-ESUS trial suggested that anticoagulation with Rivaroxaban might reduce the risk of recurrent stroke in patients with embolic stroke of undetermined stroke and PFO [22]. Dedicated studies are needed to access the role of direct oral anticoagulant agents in secondary prevention of cryptogenic stroke with PFO. Another question that still needs to be addressed is PFO closure at older ages. Data from the Oxford Vascular Study showed a high prevalence of RLS in patients who were 50 years or older. Whether this could explain some cases of cryptogenic stroke in this population, or just reaf-

Latin American Consensus of Transcranial Ultrasound and RLS firm a high incidence of PFO in general population, is still controversial [23].

RLS screening diagnostic considers the use of both TCD and transesophageal echocardiography (TEE), with advantages and disadvantages for each method [24]. The use of both exams is likely to ensure better consistency in the characterization of abnormal findings. Presence, type, and size of RLS, as well as functional and anatomic patterns observed during the Valsalva-induced or spontaneous (at-rest) detection, can provide important data for risk stratification. An effort for the standardization of the technique was recently published by the American Society of Echocardiography and Society for Cardiac Angiography and Interventions in the form of "Guidelines for the Echocardiographic Assessment of Atrial Septal Defect and PFO". These guidelines also clarify the anatomic association between PFO and atrial septum aneurysm (ASA), presence of the Chiari network (CR), and prominent Eustachian valve, and discuss other types of atrial septal defects that cannot be assessed by c-TCD. Identifying these associations to the RLS is of key clinical relevance, as it may indicate an increased risk of cerebrovascular events [25]. It is important to note that, although transthoracic echocardiography (TTE) allows the diagnosis of RLS by means of color Doppler, the flow that is inverted intermittently may not be detected, and thus TTE is not indicated for RLS diagnosis. Instead, TEE plays a central role in the evaluation of PFO both for diagnosis and for anatomical evaluation prior to choosing the closure device when indicated [26-30]. Although Valsalva maneuver is currently considered the gold standard for PFO diagnosis, some patients have poor compliance or tolerability and usually require sedation, which limits the applicability of Valsalva maneuver for characterization of RLS. Quantification of microembolic signals in such patients can be difficult and sometimes generates false negative results [24].

c-TCD has been proven to be a reliable technique and currently represents the screening test of choice for some neurology services. It is not invasive, fast, does not require patient sedation and provides reproducible results that are easy to interpret $[28,31]$. TCD uses power M-mode Doppler insonation of the basal cerebral arteries to detect microbubbles (MBs) that have crossed right-to-left into the systemic circulation. The usual technique is an insonation of middle cerebral artery (MCA), once it represents the main thromboembolic site $[32,33]$. The direct involvement of the neurologist in performing this test represents an advantage of TCD over TEE and TTE, in addition to increased patient comfort (compared with TEE), semiquantitative assessment of shunt size, and, possibly, sug- 
gests an extracardiac or intracardiac shunting [34]. Moreover, c-TCD enables improved quantification of microembolic signals, by using provocative maneuvers, as the patient is more comfortable and therefore more amenable to undergo an adequate Valsalva maneuver. Another advantage is the fact that TCD can monitor in real time the spontaneous passage of microemboli during prolonged monitorization without a provocative test, which can detect the embolic phenomenon as it takes place. Although c-TCD itself is sufficient for diagnostic screening of RLS and presents high sensitivity (97\%) and specificity (93\%) - class IIA, its use alone is not recommended [35]. The direct evaluation of RLS and anatomical observation of the atrial septum remains important [36-38]. Decision making for shunt categorization into a high-risk subgroup may depend on achieving such characterization as safely as possible. It is important to remember that detection and grading of RLS by any technique is complicated by physiologic variations. Respiratory phasic changes in right atrial pressure can result in delayed RLS and misclassification of the interatrial flow a possible intrapulmonary shunt. Elevated left atrial pressure caused by left ventricular failure, mitral stenosis, or mitral regurgitation can "prevent" RLS shunting detection, because higher right atrial pressure is required to overcome the elevated left atrial pressure. Therefore, in parallel with paradoxical embolism investigation, at least a basic TTE examination should be performed to recognize concomitant clinical conditions:

(1) ASA - present in $2-3 \%$ of the population and defined by the excursion of the septal tissue (typically, of the oval fossa) $>10 \mathrm{~mm}$ toward the right atrium or left atrium, or by a combined right-left excursion $>15 \mathrm{~mm}$. ASA may be associated with multiple septal fenestrations that may contribute to the RLS $[36,37]$.

(2) Eustachian valve and CR - remnant embryonic structures that influence the spontaneous closure of the foramen and contribute indirectly to paradoxical embolism in the presence of PFO [10]. CR is present in $2-3 \%$ of the population and, although itself benign, is associated with a higher prevalence of PFO and ASA $[36,39]$.

\section{c-TCD Technique}

Several technical details are described in the literature as important to ensure high sensitivity and specificity in c-TCD. However, protocols vary significantly, and many studies tend to use their own protocols, making it difficult to establish criteria for systematic reviews, meta-analyses, and multicenter studies to assess the predictive value of
TCD-based methods. Technical variability refers, among others, to the use of contrast agent, diameter of the venous access, unilateral or bilateral monitoring, time of testing, and timing of Valsalva maneuver. These differences may result in important disagreements regarding the outcomes of c-TCD assessments. TEE frequently uses dichotomous variables (e.g., large vs. small), whereas c-TCD has different standardizations for the quantification of the number of MBs, which can influence the estimation of RLS size, potentially precluding direct comparison between different diagnostic tests [40-47].

The main objective of this consensus statement is to establish a standardization of the c-TCD technique and its interpretation so as to improve the informative quality of the method, resulting in expanding the application of TCD in the clinical setting. The last consensus statement in this direction was published by the European Society of Neurosonology and Cerebral Hemodynamics in 2000 [48]. We have updated this statement and included new target questions.

Goals

I. To establish a standard protocol for the c-TCD examination technique that is as simple as possible, reproducible in most centers, but ensures the best sensitivity and specificity, as well as low risk for the patient.

II. To suggest a model of the c-TCD examination report that guarantees a rapid and objective interpretation of the results regarding the relationship between $\mathrm{MB}$ findings and RLS size, as well as to clarify the methodology appropriate for RLS detection at rest or after provocative maneuvers.

III. To provide a uniform characterization of the method to be used in future multicenter studies and metaanalyses.

\section{Recommendations}

The examination must be performed with the patient in the supine position and with room temperature control at $24-28^{\circ} \mathrm{C}$ to improve patient comfort and ensure adequate hemodynamics. A 2-MHz transducer with bilateral pulse, fixed onto a helmet or headband is preferentially used for the insonation of the MCA through the temporal window at a depth of 50-65 mm, using the M-mode for better evaluation. Power-M-Mode TCD might be helpful for identifying MCA and may be used equally for the test. Although not mandatory, the authors suggest multichannel device for simultaneous evaluation of both MCAs because the sensitivity of the test and the quantification of bubbles may be increased by simultaneous bilateral monitoring. 
The microembolism is identified by the difference in signal intensity between the embolic material and the arterial flow, which reflects the movement of the red blood cells. The apparatus should preferably be adjusted with a smaller sample volume $(8 \mathrm{~mm})$ and low gain, as such setting provides a better discrimination of the microembolus in the spectral analysis. The specific characteristics of the signal indicative of microembolus are unidirectionality (always in the direction of the flow in the vessel), short duration (below $0.1 \mathrm{~s}$ or $<300 \mathrm{~ms}$ ), high intensity within the Doppler flow spectrum (generally $>3$ Db above the spectral background signal), visibility at any time of the cardiac cycle (random occurrence), and presence of the typical audible sound ("chirps") $[49,50]$.

\section{Choice and Preparation of the Contrasting Agent}

Using a contrasting solution is necessary for the investigation of paradoxical embolism. The acoustic impedance of the agent is defined as the difference in density at the interface between the gaseous MBs and the surrounding tissue, with greater interface echogenicity providing better identification of MBs [51, 52]. Although some contrast agents are commercialized, it is common sense to use agitated saline solution, whose efficacy and safety have been confirmed repeatedly in the literature, especially compared to the safety and efficacy of solutions of micro-particles based on gelatin, galactose, or oxypoligelatin, which are also more expensive and less available in Latin American countries. Hereafter, the present consensus statement will discuss only the use of agitated saline solution.

The MBs generated by the agitation of the contrasting saline solution are too large to pass through the normal pulmonary vasculature and are thus easily detected. Previous comparative studies have established a high correlation regarding sensitivity and specificity between commercial contrast solutions and saline solution, and only changes to the dose of contrast agent are recommended [53]. The current consensus statement establishes as the contrast agent of choice a $10-\mathrm{mL}$ volume of injectable airsaline mixture (volumetric ratio of saline to air, 9:1, i.e., $9 \mathrm{~mL}$ of saline mixed with $1 \mathrm{~mL}$ of air) [47]. The option of mixing the contrast agent with blood from the patient remains controversial among the experts, but there was no personal report from the consensus statement signatories opposing this technique. The addition of blood to the contrast solution increases the intensity of the $\mathrm{MB}$ signal detected by echocardiography and TCD. This technical improvement would occur by the fragmentation of

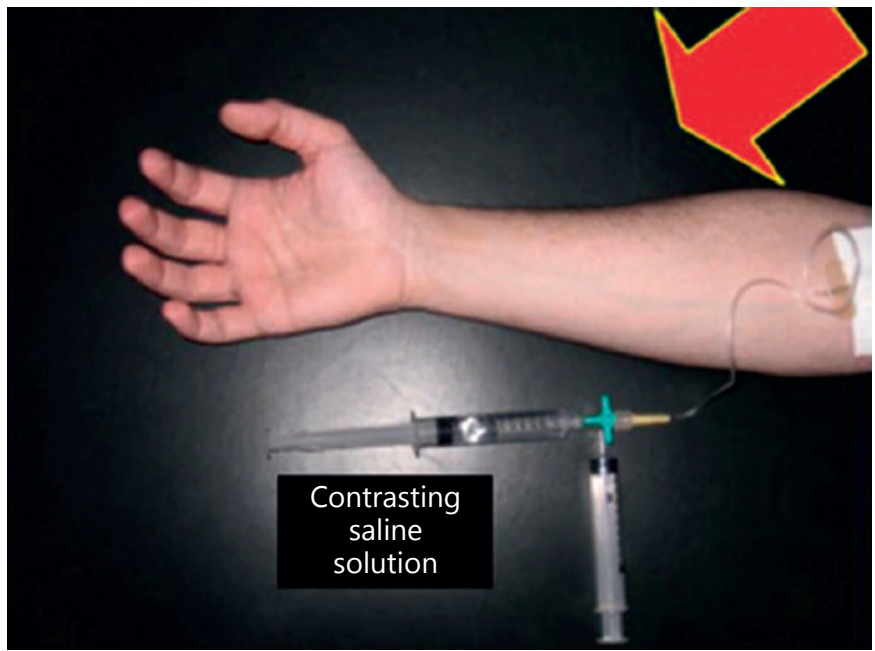

Fig. 1. Preparation of contrasting saline solution, which will be injected into a peripheral vein in the right superior limb.

the red cells, which would serve as potential enhancers for the reflection of the ultrasound $[44,46,47,52]$. Therefore, we recommend a ratio of saline to air to blood of $8: 1: 1$, that is, a $10-\mathrm{mL}$ volume of contrast agent contains $8 \mathrm{~mL}$ of saline solution, $1 \mathrm{~mL}$ of air, and $1 \mathrm{~mL}$ of the patient's blood. Mixing should be performed using a three-way stopcock connected to two 10-mL syringes (Fig. 1). The mixing should be rapid, with exchange of the contents between the syringes for at least 10 times, followed by bolus injection, observing the homogeneous ("whitish" or "pinkish") appearance of the solution to produce the MBs. Each bolus injection should contain $10 \mathrm{~mL}$ of contrasting agent.

\section{Recommendations}

I. Prepare the $\mathrm{MB}$ contrast solution by mixing $9 \mathrm{~mL}$ of saline solution and $1 \mathrm{~mL}$ of air. This should be the agent of choice (level of evidence, 1a).

II. Mixing of $1 \mathrm{~mL}$ of the patient's blood is not prohibited ( $8 \mathrm{~mL}$ saline solution $+1 \mathrm{~mL}$ air $+1 \mathrm{~mL}$ blood $)$ and can be adopted in some protocols. Blood aspiration should be performed immediately prior to shaking. Adverse events related to this protocol are rare and not significant (level of evidence, $4 \mathrm{~b}$ ).

\section{Technical Details of the c-TCD Examination}

a. Use the right arm preferentially. With the patient in supine position, extend the arm horizontally. Apply injection into the cubital vein whenever possible. 
b. Use of needle catheters (scalp or butterfly) with a needle gauge of 18-21 is preferable because such devices allow better mobility. Venous access should be used only for a short duration. There are no publications comparing the performance of non-needle (flexible) catheters.

c. If venous access is difficult and thinner needles are used, or if another location than the upper limb must be used, the examination may be performed but the variability of the technique should be described in the report (level of evidence, 4).

d. Ensure strict anti-sepsis of the puncture site. While controversies exist regarding antiseptics, iodinated alcohol and 2\% chlorhexidine remain most commonly used.

\section{Accuracy Management}

RLS can be identified better if the PFO is stretched by inducing transient or permanent pressure differences between the atria. Coughing and Valsalva maneuver are frequently used as provocative techniques, the latter being more commonly used in clinical practice and recommended by previous consensus [48]. Sedated patients may have difficulty performing either of these maneuvers. Performing a standardized protocol that includes multiple injections of agitated saline with maneuvers that temporarily increase the pressure in the right atrium can increase the diagnostic accuracy and maximize the sensitivity and specificity of the detection method [54, 55]. In patients under general anesthesia or mechanical ventilation, the Valsalva maneuver can be mimicked through ventilatory maneuvers. Elevating the patient's legs may also aid the technique. False negative results may occur due to inadequate provocative maneuvers [56]. To improve the efficacy of provocative maneuvers, it is proposed to observe a reduction of at least $25 \%$ of the mean MCA velocity, to use a manometer, or both. Patients should be instructed to maintain an expiratory pressure of $40 \mathrm{~mm} \mathrm{Hg}$ (measured using a manometer) and to avoid deep breathing before and after finishing the maneuver. The test should be repeated 3 times, and the largest number of detected MBs should be retained. Each test should take at least $1 \mathrm{~min}$, and the maneuver must be trained prior to the contrast injection to ensure better efficacy. The Valsalva maneuver should be initiated at $5 \mathrm{~s}$ after injection of the contrast agent and maintained for $10 \mathrm{~s}$, although one study showed no significant difference in measurement accuracy related to the timing of the Valsalva maneuver with respect to the injection of the contrast agent. The test with Valsalva maneuver should also be repeated 3 times. Although the repetitions might seem consuming for clinical routine, according to the authors' experience, it seems important to increase test sensitivity. In summary, the c-TCD evaluation should be performed at rest and during provocative maneuvers. If either examination reveals numerous MBs displayed in a "curtain-like" pattern, the test should be terminated and does not need to be repeated (Fig. 4).

\section{Quantification}

The quantification of MBs is considered one of the most important informational aspects of the c-TCD exam [56]. It is also relevant to consider MB quantification in each phase of the test. Risk-stratification based on c-TCD findings depends on the number of signals obtained, as well as on the phase of the exam in which the signals were obtained. It is widely accepted that larger numbers of MBs detected during the at-rest phase of the exam are indicative of the highest risk [1]. Recent TEE guidelines emphasize the need for better quantification and establishment of more uniform reporting criteria [25].

The number of MBs is affected by numerous methodological variations including dose and type of contrast agent, use of smaller veins and needles, number of tests performed, and effectiveness of provocative maneuvers, whereas most c-TCD studies initially used a 4-level classification with "shower" and "curtain" terms, the criteria have since been simplified to improve clinical acceptance and facilitate a more uniform reporting language among different examiners and protocols. The currently recognized criteria of the signal indicative of induced microembolization are as follows: unidirectional, typical visible and audible signals of short duration and high intensity, with a positive signal in the M-mode image [49].

Most authors suggest quantifying RLS size in terms of the number of MBs: up to $10 \mathrm{MBs}$, small RLS; 11-20 MBs moderate RLS; > 20 MBs, large RLS; "curtain-like" pattern, quantification is not possible because the MB signal fills the entire spectrum [57-61].

Another classification, Spencer Logarithmic Scale, considers the absence of MBs negative; the passage of 1-10 MBs grade I; $11-30 \mathrm{MBs}$ grade II; $31-100 \mathrm{MBs}$ grade III; 101-300 MBs grade IV; and above $300 \mathrm{MBs}$ grade V [62].

Although there are some variations, the consensus seems to be that RLS is present if multiple MBs are detect- 
ed $(>1)$, and is considered significant whenever $>10 \mathrm{MBs}$ are detected. Offline re-evaluation is considered for better quantification. During bilateral monitoring, the highest number obtained in each channel is what is taken into account and not the sum of these observations.

The test should, if possible, consist of 3 tests at-rest tests and 3 tests under provocative conditions. Note, however, that if "curtain-like" pattern is noted at any of these steps, the test may be terminated because the highest quantification has been established.

\section{Recommendations}

I. Always quantify the MBs in both phases, namely, at rest and during provocative maneuvers. Report separately.

II. Perform the examination 3 times at rest, and afterwards 3 times under provocative maneuvers. Consider the examination completed if the "curtain-like" pattern is observed in any of these steps (Fig. 4).

III. Explain the provocative maneuver to the patient and have them practice it prior to beginning the test in order to check that the provocative test has a reliable response.

IV. Consider it a positive result the detection of at least one $\mathrm{MB}$ with spectral visualization and coincident signal on M-mode, as well the typical sound pattern. Consider it a non-significant positive result if fewer than $10 \mathrm{MBs}$ are detected only during the provocative maneuver.

V. Report the findings according to 4 categorization levels: no MBs, negative test for RLS; $\leq 10 \mathrm{MBs}$ (Fig. 2); small or non-significant RLS; $>10$ but $<20 \mathrm{MBs,} \mathrm{moderate} \mathrm{RLS}$ (Fig. 3); $\geq 20 \mathrm{MBs}$ and no "curtain-like" pattern, large RLS; "curtain pattern", uncountable MBs (Fig. 4). The shorter report is an option.

VI. For bilateral tests, use the highest number obtained in each channel and do not sum the number of MBs detected in each channel.

\section{False-Positive and False-Negative Results}

Delayed detection of MBs in the MCAs ( $>10$ s or $>10$ cardiac cycles) may be corroborative of intrapulmonary RLS, as observed in arteriovenous malformations that allow embolic passage through the capillaries. However, in practice, there is a significant overlap between the time of $\mathrm{MB}$ appearance when originating from a cardiac shunt such as PFO or originating from an intrapulmonary RLS. Therefore, time should not represent a reliable indicator of RLS location. Instead, the delay is significantly influenced by the size and intensity of the RLS flow. Although

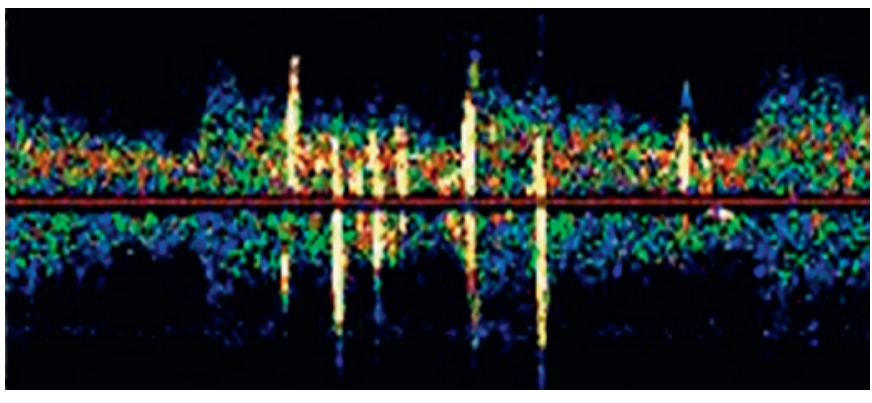

Fig. 2. 1-10 MBs (small shunt).

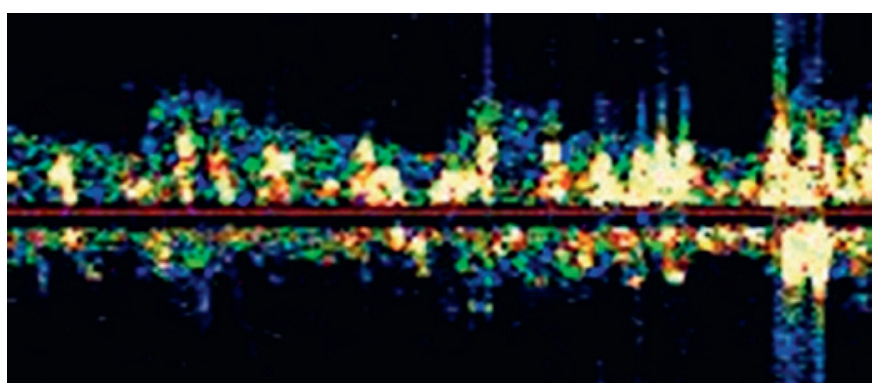

Fig. 3. 11-20 MBs (moderate shunt).

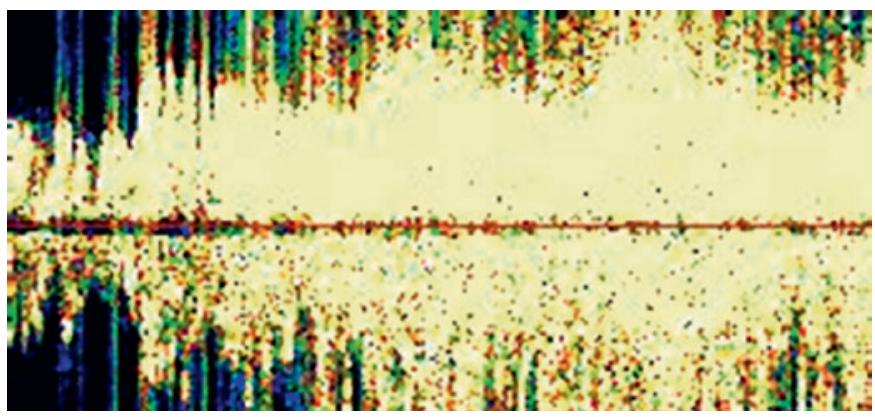

Fig. 4. "Curtain" pattern.

TCD and TEE can help determine the location of the RLS, it is not possible to safely confirm this location, and performing contrast tests of the pulmonary circulation can be indicated when there is significant divergence in observations [34, 63].

\section{Report Recommendation}

The inclusion of printed screenshots in the c-TCD report is highly recommended. The image should preferably contain the largest number of MBs detected, and the number of channels used should be mentioned. 


\section{Concluding Remarks}

TEE is considered the gold standard for the diagnosis of cardiac RLS. The advantage of direct visualization of the atrial septum allows better anatomical characterization, which is essential for PFO diagnosis. c-TCD represents an alternative method for RLS detection and is undoubtedly an excellent screening test because it provides high sensitivity and specificity. The advantages of c-TCD include better patient comfort and improved quantitative assessment of RLS size by allowing to combine the use of provocative maneuvers with the ability to identify embolic phenomena in action through real-time monitoring, which adds an important functional impact to the diagnosis. Thus, TCD and TEE with MB contrast can be considered complementary techniques in the evaluation of RLS.

The role of RLS in the occurrence of cerebrovascular events remains controversial, but there is no doubt that its presence should be considered a risk factor in several clinical situations. It is important to note that rarely paradoxical embolism can also be caused by RLS at the pulmonary level due to pulmonary arteriovenous fistula. A cortical lesion is suggestive of embolic etiology and it should be remembered. The diagnosis of RLS in cryptogenic stroke increases the possibility of paradoxical embolism, even without the knowledge of the source, but careful and individualized evaluation of the clinical context is paramount. Repeated investigation of paroxysmal atrial fibrillation has been relevant and attention to differential diagnosis even in the presence of RLS should be considered. We strongly recommend a heart-brain team to individualize a patient with $\mathrm{PFO}$, using personal information, neuroimaging findings, anatomical (ETE), and functional characteristics of PFO (c-TCD) to decide the best strategy of treatment.

\section{Summary of Recommended Procedures (Protocol Example)}

I. Use supine position, intravenous access preferably in the right upper limb, using an 18-21-gauge needle catheter.

II. Monitor both MCAs with the device affixed to a helmet whenever possible.

III. Use venous access in a cubital vein connected to the tubing using a three-way stopcock.

IV. In a $10-\mathrm{mL}$ syringe connected to the stopcock, combine $8 \mathrm{~mL}$ of saline solution with $1 \mathrm{~mL}$ of the patient's blood and $1 \mathrm{~mL}$ of air. Some laboratories avoid using the patient's blood in the contrast solution, in which case $9 \mathrm{~mL}$ of saline solution is mixed with $1 \mathrm{~mL}$ of air.

$\mathrm{V}$. Mix the contrasting solution vigorous between the syringes for at least 10 times until the rosacea or whitish appearance of the solution is observed. Inject immediately.

VI. Repeat the test 3 times at rest and 3 times under provocative maneuvers, unless "curtain-like" pattern of MBs is detected at any step of the test.

VII. Provocative maneuvers should be monitored to ensure effectiveness. Whenever possible, use a manometer that enables flow velocity control (reduction of at least $25 \%$ observed in the spectral curve, followed by a corresponding increase). The duration of the Valsalva maneuver should be $10 \mathrm{~s}$.

VIII. Report quantitative results according to 4 categorization levels: no MBs, negative test for RLS; $\leq 10 \mathrm{MBs,}$ small or non-significant RLS (Fig. 2); $>10$ but $<20 \mathrm{MBs,}$ moderate RLS (Fig. 3); $\geq 20$ MBs and no "curtain-like" pattern, large RLS; "curtain pattern", uncountable MBs (Fig. 4; no possibility of discriminating MB signals in the spectrum). It is possible to shorten the report using negative, significant $>10$ and "curtain" (Fig. 2-4).

IX. Retain and report the highest number of MBs observed for each channel and not the sum of the values recorded for each channel.

$\mathrm{X}$. Draft the report with as much detail as possible, emphasizing the outcome of MB quantification especially when fewer than $10 \mathrm{MBs}$ were found, indicating non-significant RLS. Specify whether the results were obtained at rest or only after a provocative maneuver.

XI. Indicate if spontaneous microemboli during the test for RLS were also detected. Be clear to avoid misinterpretation regarding microembolization activity.

XII. Provide an explicit description of any technical variation that had to be applied in order to perform the evaluation (as insonation of vertebral or basilar artery).

XIII. Include the printed screenshot in the report, preferably including an image that shows the number of channels and the largest number of MBs detected.

\section{Acknowledgement}

Neurosonology Research Group of the World Federation of Neurology.

\section{Statement of Ethics}

The authors have no ethical conflicts to disclose. 


\section{Disclosure Statement}

The authors have no conflicts of interest to declare.

\section{Funding Sources}

The authors have no funding to report.

\section{Author Contributions}

V.F.Z., V.C.S., and R.B.: writing and organizing discussion meetings. M.C.L., R.B., G.P.B., A.C.C.B.L., S.R.C.F., O.M.P.N., C.H.C.M., G.S., M.A.M.A., D.L.G., E.B.-S.-S., M.L.O., P.A.P.J., J.O.-F., W.M.A., G.R.F., C.P., S.V.C., and A.B.: participation in discussion meetings. A.M., N.M.B., A.R., and M.D.S.: review of the text and critical analysis.

\section{References}

1 Mas JL, Derumeaux G, Guillon B, Massardier E, Hosseini H, Mechtouff L, et al.; CLOSE Investigators. Patent Foramen Ovale Closure or Anticoagulation vs. Antiplatelets after Stroke. N Engl J Med. 2017 Sep;377(11):1011-21.

2 Saver JL, Carroll JD, Thaler DE, Smalling RW, MacDonald LA, Marks DS, et al.; RESPECT Investigators. Long-Term Outcomes of Patent Foramen Ovale Closure or Medical Therapy after Stroke. N Engl J Med. 2017 Sep; 377(11):1022-32.

3 Søndergaard L, Kasner SE, Rhodes JF, Andersen G, Iversen HK, Nielsen-Kudsk JE, et al.; Gore REDUCE Clinical Study Investigators. Patent Foramen Ovale Closure or Antiplatelet Therapy for Cryptogenic Stroke. N Engl J Med. 2017 Sep;377(11):1033-42.

4 Dao CN, Tobis JM. PFO and paradoxical embolism producing events other than stroke. Catheter Cardiovasc Interv. 2011 May;77(6): 903-9.

5 Riaz IB, Dhoble A, Mizyed A, Hsu CH, Husnain $\mathrm{M}$, Lee JZ, et al. Transcatheter patent foramen ovale closure versus medical therapy for cryptogenic stroke: a meta-analysis of randomized clinical trials. BMC Cardiovasc Disord. 2013 Dec;13(1):116.

6 Bang OY, Lee MJ, Ryoo S, Kim SJ, Kim JW. Patent Foramen Ovale and Stroke-Current Status. J Stroke. 2015 Sep;17(3):229-37.

7 Rigatelli G, Aggio S, Cardaioli P, Braggion G, Giordan M, Dell'avvocata F, et al. Left atrial dysfunction in patients with patent foramen ovale and atrial septal aneurysm: an alternative concurrent mechanism for arterial embolism? JACC Cardiovasc Interv. 2009 Jul;2(7):655-62.

8 Song BG, Park SW, Lee SC, Choi JO, Park SJ, Chang SA, et al. Real-time 3D TEE for multiperforated interatrial septum. JACC Cardiovasc Imaging. 2010 Nov;3(11):1199.

9 Pushparajah K, Miller OI, Simpson JM. 3D echocardiography of the atrial septum: anatomical features and landmarks for the echocardiographer. JACC Cardiovasc Imaging. 2010 Sep;3(9):981-4.

10 Amarenco P, Bogousslavsky J, Caplan LR, Donnan GA, Wolf ME, Hennerici MG. The ASCOD phenotyping of ischemic stroke (Updated ASCO Phenotyping). Cerebrovasc Dis. 2013;36(1):1-5

11 Furlan AJ, Reisman M, Massaro J, Mauri L, Adams H, Albers GW, et al.; CLOSURE I In- vestigators. Closure or medical therapy for cryptogenic stroke with patent foramen ovale. N Engl J Med. 2012 Mar;366(11):991-9.

12 Meier B, Kalesan B, Mattle HP, Khattab AA, Hildick-Smith D, Dudek D, et al.; PC Trial Investigators. Percutaneous closure of patent foramen ovale in cryptogenic embolism. N Engl J Med. 2013 Mar;368(12):1083-91.

13 Carroll JD, Saver JL, Thaler DE, Smalling RW, Berry S, MacDonald LA, et al.; RESPECT Investigators. Closure of patent foramen ovale versus medical therapy after cryptogenic stroke. N Engl J Med. 2013 Mar;368(12):1092100.

14 Hart RG, Diener HC, Coutts SB, Easton JD, Granger CB, O’Donnell MJ, et al.; Cryptogenic Stroke/ESUS International Working Group. Embolic strokes of undetermined source: the case for a new clinical construct. Lancet Neurol. 2014 Apr;13(4):429-38.

15 Kent DM, Ruthazer R, Weimar C, Mas JL, Serena J, Homma S, et al. An index to identify stroke-related vs incidental patent foramen ovale in cryptogenic stroke. Neurology. 2013 Aug;81(7):619-25.

16 Rigatelli G, Dell'Avvocata F, Giordan M, Braggion G, Aggio S, Chinaglia M, et al. Embolic implications of combined risk factors in patients with patent foramen ovale (the CARPE criteria): consideration for primary prevention closure? J Interv Cardiol. 2009 Aug;22(4):398-403.

17 Ropper AH. Tipping Point for Patent Foramen Ovale Closure. N Engl J Med. 2017 Sep; 377(11):1093-5.

18 Farb A, Ibrahim NG, Zuckerman BD. Patent Foramen Ovale after Cryptogenic Stroke - Assessing the Evidence for Closure. N Engl J Med. 2017 Sep;377(11):1006-9.

19 Zhang XL, Kang LN, Wang L, Xu B. Percutaneous closure versus medical therapy for stroke with patent foramen Ovale: a systematic review and meta-analysis. BMC Cardiovasc Disord. 2018 Mar;18(1):45.

20 Rigatelli G, Dell'avvocata F, Daggubati R, Dung HT, Nghia NT, Nanjiundappa A, et al. Impact of interatrial septum anatomic features on short- and long-term outcomes after transcatheter closure of patent foramen ovale: single device type versus anatomic-driven device selection strategy. J Interv Cardiol. 2013 Aug;26(4):392-8.
21 Cooke JC, Gelman JS, Harper RW. Chiari network entanglement and herniation into the left atrium by an atrial septal defect occluder device. J Am Soc Echocardiogr. 1999 Jul; 12(7):601-3.

22 Kasner SE, Swaminathan B, Lavados P, Sharma M, Muir K, Veltkamp R, et al.; NAVIGATE ESUS Investigators. Rivaroxaban or aspirin for patent foramen ovale and embolic stroke of undetermined source: a prespecified subgroup analysis from the NAVIGATE ESUS trial. Lancet Neurol. 2018 Dec;17(12): 1053-60.

23 Mazzucco S, Li L, Binney L, Rothwell PM; Oxford Vascular Study Phenotyped Cohort. Prevalence of patent foramen ovale in cryptogenic transient ischaemic attack and non-disabling stroke at older ages: a population-based study, systematic review, and meta-analysis. Lancet Neurol. 2018 Jul;17(7):609-17.

24 Souteyrand G, Motreff P, Lusson JR, Rodriguez R, Geoffroy E, Dauphin C, et al. Comparison of transthoracic echocardiography using second harmonic imaging, transcranial Doppler and transesophageal echocardiography for the detection of patent foramen ovale in stroke patients. Eur J Echocardiogr. 2006 Mar;7(2):147-54.

25 Silvestry FE, Cohen MS, Armsby LB, Burkule NJ, Fleishman CE, Hijazi ZM, et al.; American Society of Echocardiography; Society for Cardiac Angiography and Interventions. Guidelines for the Echocardiographic Assessment of Atrial Septal Defect and Patent Foramen Ovale: From the American Society of Echocardiography and Society for Cardiac Angiography and Interventions. J Am Soc Echocardiogr. 2015 Aug;28(8):910-58.

26 Homma S, Sacco RL. Patent foramen ovale and stroke. Circulation. 2005 Aug;112(7): 1063-72.

27 Agmon Y, Khandheria BK, Meissner I, Gentile F, Whisnant JP, Sicks JD, et al. Frequency of atrial septal aneurysms in patients with cerebral ischemic events. Circulation. 1999 Apr; 99(15):1942-4.

28 Giannopoulos A, Gavras C, Sarioglou S, Agathagelou F, Kassapoglou I, Athanassiadou F. Atrial septal aneurysms in childhood: prevalence, classification, and concurrent abnormalities. Cardiol Young. 2014 Jun;24(3):4538.
Latin American Consensus of Transcranial Ultrasound and RLS
Cerebrovasc Dis 2019;48:99-108 DOI: $10.1159 / 000503851$ 
29 Tanaka J, Izumo M, Fukuoka Y, Saitoh T, Harada K, Harada K, et al. Comparison of two-dimensional versus real-time three-dimensional transesophageal echocardiography for evaluation of patent foramen ovale morphology. Am J Cardiol. 2013 Apr;111(7): 1052-6.

30 Rana BS, Shapiro LM, McCarthy KP, Ho SY. Three-dimensional imaging of the atrial septum and patent foramen ovale anatomy: defining the morphological phenotypes of patent foramen ovale. Eur J Echocardiogr. 2010 Dec;11(10):i19-25.

31 Lange MC, Zétola VF, de Souza AM, Piovesan EJ, Muzzio JA, Germiniani FM, et al. Transcranial Doppler for patent foramen ovale screening: is there a good correlation with transesophageal echocardiography? Arq Neuropsiquiatr. 2008 Dec;66(4):785-9.

32 Sloan MA, Alexandrov AV, Tegeler $\mathrm{CH}$, Spencer MP, Caplan LR, Feldmann E, et al. Assessment: transcranial Doppler ultrasonography: report of the Therapeutics and Technology Assessment Subcommittee of the American Academy of Neurology. Neurology. 2004 May;62(9):1468-81.

33 Caputi L, Carriero MR, Falcone C, Parati E, Piotti P, Materazzo C, et al. Transcranial Doppler and transesophageal echocardiography: comparison of both techniques and prospective clinical relevance of transcranial Doppler in patent foramen ovale detection. J Stroke Cerebrovasc Dis. 2009 Sep-Oct;18(5):343-8.

34 Lange MC, Zétola VF, deSouza AM, Novak FM, Piovesan EJ, Werneck LC. Intracranial embolism characteristics in PFO patients: a comparison between positive and negative PFO by transesophageal echocardiography: the rule of nine. J Neurol Sci. 2010 Jun;293(12):106-9.

35 Mojadidi MK, Roberts SC, Winoker JS, Romero J, Goodman-Meza D, Gevorgyan R, et al. Accuracy of transcranial Doppler for the diagnosis of intracardiac right-to-left shunt: a bivariate meta-analysis of prospective studies. JACC Cardiovasc Imaging. 2014 Mar;7(3): 236-50.

36 Schneider B, Hofmann T, Justen MH, Meinertz T. Chiari's network: normal anatomic variant or risk factor for arterial embolic events? J Am Coll Cardiol. 1995 Jul;26(1): 203-10.

37 Schuchlenz HW, Saurer G, Weihs W, Rehak P. Persisting eustachian valve in adults: relation to patent foramen ovale and cerebrovascular events. J Am Soc Echocardiogr. 2004 Mar;17(3):231-3.

38 Scaffa R, Spaziani C, Leporace M, Leonetti S, Di Roma M, Gaspardone A, et al. Voluminous atrial septal aneurysm may mask a large double atrial septal defect. Ann Thorac Surg. 2012 Feb;93(2):e41.

39 Marriott K, Manins V, Forshaw A, Wright J, Pascoe R. Detection of right-to-left atrial communication using agitated saline contrast imaging: experience with 1162 patients and recommendations for echocardiography. J
Am Soc Echocardiogr. 2013 Jan;26(1):96102.

40 Mas JL, Arquizan C, Lamy C, Zuber M, Cabanes L, Derumeaux G, et al.; Patent Foramen Ovale and Atrial Septal Aneurysm Study Group. Recurrent cerebrovascular events associated with patent foramen ovale, atrial septal aneurysm, or both. N Engl J Med. 2001 Dec;345(24):1740-6.

41 Di Tullio M, Sacco RL, Venketasubramanian N, Sherman D, Mohr JP, Homma S. Comparison of diagnostic techniques for the detection of a patent foramen ovale in stroke patients. Stroke. 1993 Jul;24(7):1020-4.

42 Attaran RR, Ata I, Kudithipudi V, Foster L, Sorrell VL. Protocol for optimal detection and exclusion of a patent foramen ovale using transthoracic echocardiography with agitated saline microbubbles. Echocardiography. 2006 Aug;23(7):616-22.

43 Johansson MC, Helgason H, Dellborg M, Eriksson P. Sensitivity for detection of patent foramen ovale increased with increasing number of contrast injections: a descriptive study with contrast transesophageal echocardiography. J Am Soc Echocardiogr. 2008 May; 21(5):419-24.

44 Fan S, Nagai T, Luo H, Atar S, Naqvi T, Birnbaum Y, et al. Superiority of the combination of blood and agitated saline for routine contrast enhancement. J Am Soc Echocardiogr. 1999 Feb;12(2):94-8.

45 Woods TD, Patel A. A critical review of patent foramen ovale detection using saline contrast echocardiography: when bubbles lie. J Am Soc Echocardiogr. 2006 Feb;19(2):215-22.

46 Johansson MC, Eriksson P, Guron CW, Dellborg M. Pitfalls in diagnosing PFO: characteristics of false-negative contrast injections during transesophageal echocardiography in patients with patent foramen ovales. $J$ Am Soc Echocardiogr. 2010 Nov;23(11):1136-42.

47 Lange MC, Zétola VF, Piovesan ÉJ, Werneck LC. Saline versus saline with blood as a contrast agent for right-to-left shunt diagnosis by transcranial Doppler: is there a significant difference? J Neuroimaging. 2012 Jan;22(1):1720.

48 Jauss M, Zanette E. Detection of right-to-left shunt with ultrasound contrast agent and transcranial Doppler sonography. Cerebrovasc Dis. 2000 Nov-Dec;10(6):490-6.

49 Ringelstein EB, Droste DW, Babikian VL, Evans DH, Grosset DG, Kaps M, et al.; International Consensus Group on Microembolus Detection. Consensus on microembolus detection by TCD. Stroke. 1998 Mar;29(3):725-9.

50 Markus H. Importance of time-window overlap in the detection and analysis of embolic signals. Stroke. 1995 Nov;26(11):2044-7.

51 Shi WT, Forsberg F, Raichlen JS, Needleman L, Goldberg BB. Pressure dependence of subharmonic signals from contrast microbubbles. Ultrasound Med Biol. 1999 Feb;25(2): 275-83.

52 Gentile M, De Vito A, Azzini C, Tamborino C, Casetta I. Adding blood to agitated saline significantly improves detection of right-toleft shunt by contrast-transcranial color-coded duplex sonography. Ultrasound Med Biol. 2014 Nov;40(11):2637-41.

53 Puledda F, Toscano M, Pieroni A, Veneroso G, Di Piero V, Vicenzini E. Right-to-left shunt detection sensitivity with air-saline and airsuccinil gelatin transcranial Doppler. Int J Stroke. 2016 Feb;11(2):229-38.

54 Lange MC, Zétola VF, Piovesan EJ, Werneck LC. Valsalva maneuver procedures in the diagnosis of right-to-left shunt by contrast-enhanced transcranial doppler using agitated saline solution with blood as a contrast agent. Arq Neuropsiquiatr. 2010 Jun;68(3):410-3.

55 Guo YZ, Gao YS, Guo ZN, Niu PP, Yang Y, Xing YQ. Comparison of Different Methods of Valsalva Maneuver for Right-to-left Shunt Detection by Contrast-Enhanced Transcranial Doppler. Ultrasound Med Biol. 2016 May;42(5):1124-9.

56 Rodrigues AC, Picard MH, Carbone A, Arruda AL, Flores T, Klohn J, et al. Importance of adequately performed Valsalva maneuver to detect patent foramen ovale during transesophageal echocardiography. J Am Soc Echocardiogr. 2013 Nov;26(11):1337-43.

57 Tobe J, Bogiatzi C, Munoz C, Tamayo A, Spence JD. Transcranial Doppler is Complementary to Echocardiography for Detection and Risk Stratification of Patent Foramen Ovale. Can J Cardiol. 2016 Aug;32(8):986.e9-16.

58 Rigatelli G, Dell'Avvocata F, Cardaioli P, Giordan M, Braggion G, Aggio S, et al. Permanent right-to-left shunt is the key factor in managing patent foramen ovale. J Am Coll Cardiol. 2011 Nov;58(21):2257-61.

59 González-Alujas T, Evangelista A, Santamarina E, Rubiera M, Gómez-Bosch Z, Rodríguez-Palomares JF, et al. Diagnosis and quantification of patent foramen ovale. Which is the reference technique? Simultaneous study with transcranial Doppler, transthoracic and transesophageal echocardiography. Rev Esp Cardiol. 2011 Feb;64(2):133-9.

60 Benjamin S. Wessler, David M. Kent, David E. Thaler, Robin Ruthazer, Jennifer S. Lutz, Joaquín Serena. The RoPE Score and Rightto-Left Shunt Severity by Transcranial Doppler in the CODICIA Study. Cerebrovasc Dis. 2015;40(0):52-8.

61 D'Andrea A, Conte M, Riegler L, Scarafile R, Cocchia R, Pezzullo E, et al. Transcranial Doppler Ultrasound: Incremental Diagnostic Role in Cryptogenic Stroke Part II. J Cardiovasc Echogr. 2016 Jul-Sep;26(3):71-7.

62 Lao AY, Sharma VK, Tsivgoulis G, Frey JL, Malkoff MD, Navarro JC, et al. Detection of right-to-left shunts: comparison between the International Consensus and Spencer Logarithmic Scale criteria. J Neuroimaging. 2008 Oct; 18(4):402-6.

63 Guchlerner M, Kardos P, Liss-Koch E, Franke $\mathrm{J}$, Wunderlich N, Bertog S, et al. PFO and right-to-left shunting in patients with obstructive sleep apnea. J Clin Sleep Med. 2012 Aug;8(4):375-80. 